\title{
OBITUARIO
}

\section{In Memoriam doctor Hernando De Macedo Ruiz (1925-2015)}

\section{In Memoriam doctor Hernando De Macedo Ruiz (1925-2015)}

\section{Irma Franke}

Museo de Historia Natural, Universidad Nacional Mayor de San Marcos. Apartado Postal 14-0434, Lima 14, Perú. Email Irma Franke: irma.franke@gmail.com

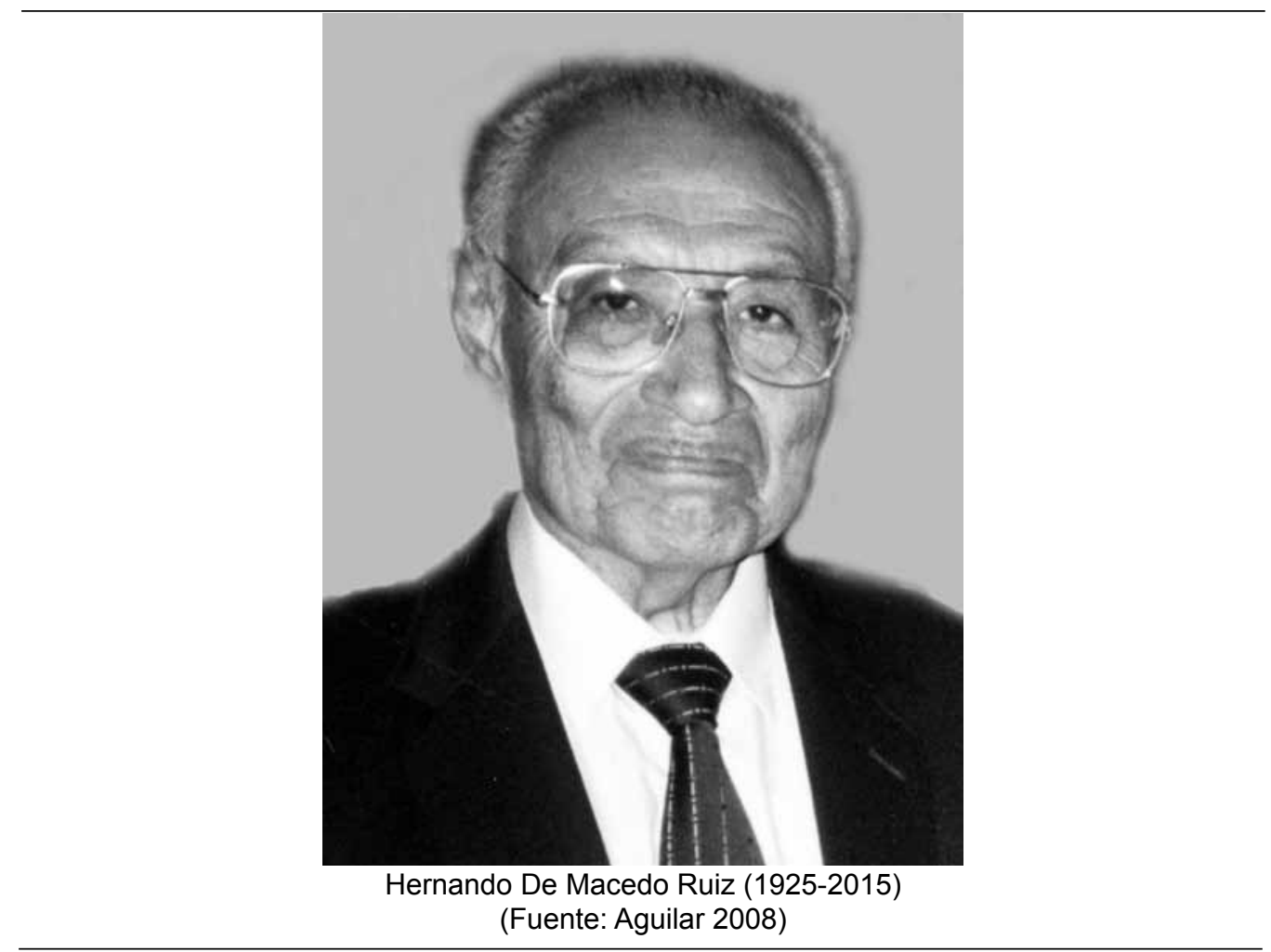

Celso Hernando De Macedo Ruiz nació en Lima el 16 de marzo de 1925 en la familia formada por don Celso De Macedo Pastor y dońa Aurora Ruiz de De Macedo. Fue el mayor de tres hermanos, dos de los cuales, Humfredo y Elia, le sobreviven. Fue nieto del médico Dr. Carlos Molares Macedo, segundo Director del Museo de Historia Natural (1938 a 1946) y también catedrático de Biología General en la Facultad de Ciencias de la Universidad Nacional Mayor de San Marcos (Aguilar 1998b).

\section{Citación:}

Franke I.. 2015. In Memoriam doctor Hernando de Macedo Ruiz (19252015). Revista peruana de biología 22(2): 125 - 134 (Octubre 2015). doi: http://dx.doi.org/10.15381/rpb.v22i2.11348 


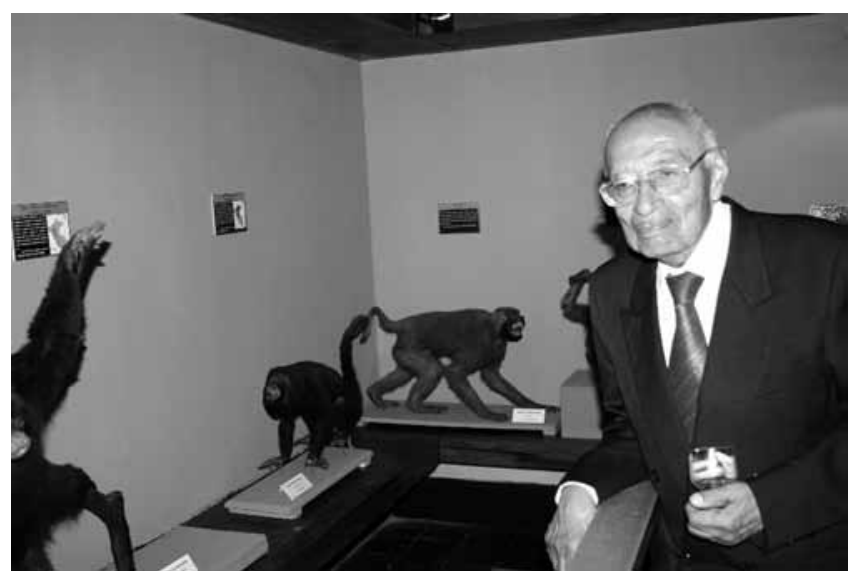

Dr. De Macedo en la remodelación de la Exhibición de Mamíferos del Museo de Historia Natural, 28 de febrero del 2011 (Foto V. Pacheco).

Su niñez transcurrió principalmente en Puno, donde la familia tenía una hermosa casa, y en la ciudad de Arequipa donde también tenían otra residencia y en la Hacienda Checayani, esta última propiedad muy ligada a muchos aspectos de su vida. Para permanecer más cerca a su familia, que radicaba principalmente en la hacienda, sus estudios escolares de primaria los realizó en el Colegio La Salle de Arequipa. Los estudios de secundaria los realizó en el Colegio La Salle de Lima, terminándolos en 1941. Durante toda la secundaria fue compañero de carpeta del padre del ornitólogo y conservacionista M. Rosina (M. Rosina com pers).

\section{Universidad de San Marcos, Grados y Títulos}

En 1945, el Dr. De Macedo ingresó a la Escuela de Ciencias Biológicas de la Facultad de Ciencias de la Universidad Nacional Mayor de San Marcos. Ese ańo ingresaron 25 estudiantes a la facultad de Ciencias, aunque sólo siete se orientaron a los distintos campos de la Biología. Entre ellos se encontraban Oscar Tovar, Javier Ortiz de la Puente y Pedro Aguilar, todos ellos futuros profesores de San Marcos. En los años 40 los estudios de Biología duraban 4 años y se dictaban principalmente en la casona del Parque Universitario. En 1945 el único curso que se dictaba en el Museo de Historia Natural era la teoría de Zoología General, a cargo de un joven y exigente profesor, Luis GonzalesMugaburu Figueroa. Las clases tenían lugar en el auditorio del museo y el profesor tenía la costumbre de exigir que los alumnos se levantaran al momento de su ingreso, que lo saludaran con un fuerte "Buenos Días", para luego pasar a llamar lista y tomar paso oral a los que iban confirmando su presencia. Esto generó que muchos no contestaran al ser llamados para eludir el paso oral y otros llegaran al museo antes de la hora de clase, que tenía el horario de 11 a 13 horas, para prepararse. Aquellos que llegaban temprano con frecuencia encontraban los esquemas dibujados en la pizarra por Hernando De Macedo, quien mencionaba que era un permanente ayudante "ad-honorem", "a título gracioso" como él solía decir. De Macedo y Aguilar repasaban con las maquetas de los diferentes grupos animales y algunas preparaciones de las exhibiciones en el museo pues los exámenes de zoología general eran generalmente orales y muy exigentes (Aguilar 1998a).

En 1946, casi todos los cursos de zoología para los estudiantes de la Escuela de Ciencias Biológicas pasaron a ser dictados en el Museo de Historia Natural en ambientes acondicionados en el sótano (Aguilar 1998a). La gran cantidad de tiempo que pasaban Hernando De Macedo y sus compañeros en el Museo de Historia Natural estableció en ellos vínculos muy fuertes con esta institución que se extendieron bastante más allá de 1948, ańo en que terminaron sus estudios y para el Dr. De Macedo continuarían hasta su jubilación.

Luego de terminar sus estudios, Hernando De Macedo realizó un estudio de ranas del género Batrachophrynus, asesorado por el Dr. Jehan Vellard, herpetólogo que en ese momento era Director del Museo de Historia Natural. Este trabajo contiene observaciones sobre el comportamiento en su ambiente natural de la rana de Junín (ahora casi extinta) que son inéditas, minuciosas y exquisitamente redactadas (César Aguilar, com pers.). En 1950 sustentó este trabajo teniendo como miembro de su Jurado al Dr. Hans-Wilhelm Koepcke, obteniendo el grado de Bachiller en Ciencias Biológicas (De Macedo 1950). Los títulos y grados superiores los dilató por diversas circunstancias. En 1970 obtuvo el Título Profesional de Biólogo y en 1976 el grado de Doctor en Ciencias Biológicas (De Macedo 1970), ambos de la Universidad Nacional Mayor de San Marcos.

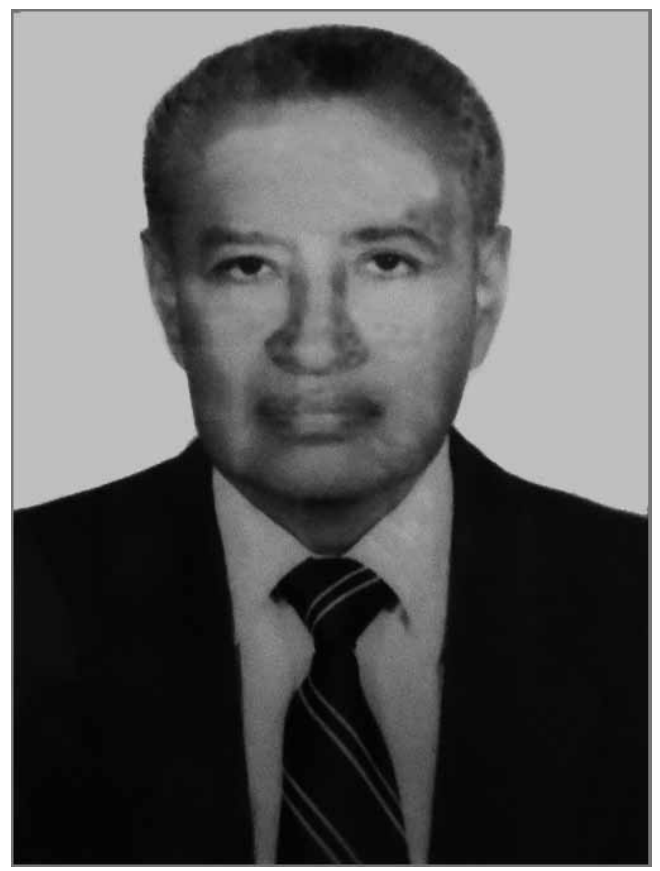

Hernando De Macedo Ruiz, en los años 1960. (Foto I. Franke)

\section{Estudios e investigaciones en el extranjero}

En agosto de 1950, Hernando De Macedo viajó a Estados Unidos para ampliar sus conocimientos, llevando como alumno libre algunas materias en el Iowa State College, Ames, Ia. En 1952, al culminar estos cursos regresó al Perú.

En 1958-1959 realizó estudios de Anatomía Comparada en la Universidad Chtistian Albrecht de Kiel, Alemania mediante una beca que había obtenido de la Fundación Alexander von Humboldt.

En 1959 realizó investigaciones en ornitología en Francia, en la Estación Biológica Tour du Valat, Arles, Bouches du Rhone, y en el Museo de Historia Natural de Paris como becario del gobierno francés. 


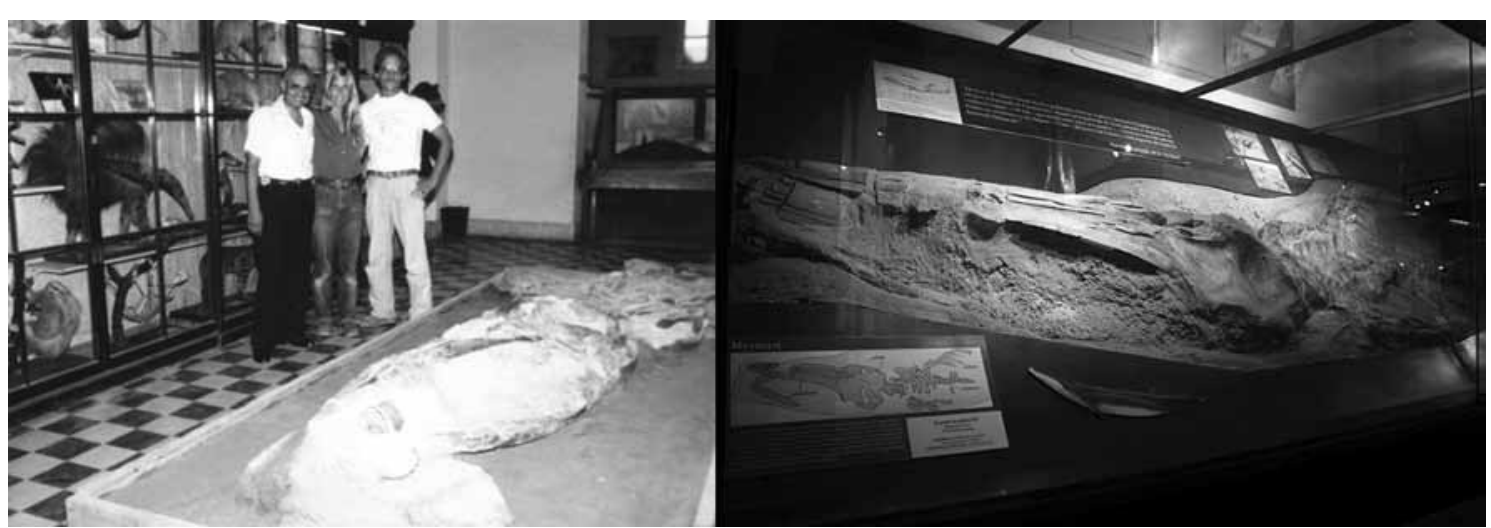

Preparación de la exhibición del primer fósil de ballena en el Museo de Historia Natural (1980's) (Foto R. Salas) y la exhibición actual (Foto J. Tejada)

Como resultado de estas visitas a Estados Unidos, Alemania y Francia, sus conocimientos de idiomas eran excelentes. En una conversión podía alternar con la mayor soltura pasando del castellano al inglés, luego al francés y al alemán, para regresar al castellano (Larson com.pers.). El conocimiento de idiomas le permitió tener una excelente biblioteca y ser miembro de numerosas instituciones nacionales y extranjeras, como la Sociedad de Fauna y Flora de París, la Sociedad Alemana de Mastozoología (con sede en Berlín) y la Sociedad Geográfica de Lima, entre otras.

\section{La Estación Altoandina de Biología de Checayani}

Durante el periodo 1950-1952 en que realizó estudios en Estados Unidos, concibió la idea de fundar una estación biológica en la propiedad familiar de Checayani. Esta hacienda, ubicada en el distrito de Muñani, provincia de Azángaro, departamento de Puno, había pertenecido al político, abogado y orador peruano José Domingo Choquehuanca. En los inicios de la República, el Estado confiscó esta propiedad por deudas al erario. Posteriormente, el "Soberano Congreso General Constituyente" asignó la "Estancia Checayani” al general Juan Crisóstomo Torrico, como fracción del premio que le correspondía en su calidad de integrante de los "Vencedores de Ancash" (Yungay). En setiembre de 1841, el mencionado general vendió la propiedad al Sr. Mariano Riquelme, antepasado materno de Hernando De Macedo.

Desde mediados de 1952 se acondicionó una habitación en la casa hacienda, dotándola de material e instrumentos de laboratorio, además de un grupo electrógeno sistema Diesel. El 2 de diciembre de 1953 se fundó oficialmente la Estación Altoandina de Biología (Aguilar 1998b). La estación estaba ubicada a 3900 $\mathrm{m}$ rodeada de colinas que alcanzaban $4200 \mathrm{~m}$, con planicies altoandinas y montańas de $4600 \mathrm{~m}$. Esta zona contiene los diversos hábitats característicos de la zona altoandina del sur del Perú, dominada por pajonal de puna y entre los que destacan los rodales de Puya y bosques de Polylepis (Dorst 1962). Como primer investigador fue invitado el Dr. Jehan Vellard, en ese momento director del Museo de Historia Natural, quien realizó un estudio que se centró en el veneno de Tachymenis peruviana, único ofidio de la región (Vellard 1955).

El reconocimiento oficial de la Estación Altoandina de Biología (USAID 1966) tuvo lugar el 25 de marzo de 1954 mediante el oficio V.100-1 firmado por el Dr. Cristóbal Vecorena Linares, entonces Director de Pesquería y Caza del Ministerio de Agricultura. Posteriormente, dada la preocupación por la fauna y flora dentro del ámbito del fundo Checayani se modificó el nombre en 1968 a Estación Altoandina de Biología y Reserva Zoobotánica de Checayani, nombre con el que apareció desde 1968 en el directorio de instituciones científicas The World of Learning.

Las vinculaciones de Hernando De Macedo con la Estación Biológica Casa Humbolt fundada por María y Hans-Wilhelm Koepcke propiciaron la afluencia de científicos de diversas nacionalidades. Entre los investigadores que realizaron estudios en Checayani se pueden mencionar a Jean Dorst, Director del Museo de Historia Natural de París, Friederich Schaller, Director del Instituto Zoológico de Viena y Wolf Herre, fundador y director del Instituto para estudios sobre animales domésticos en Kiel, Alemania. Este último gestionaría la beca para su estadía

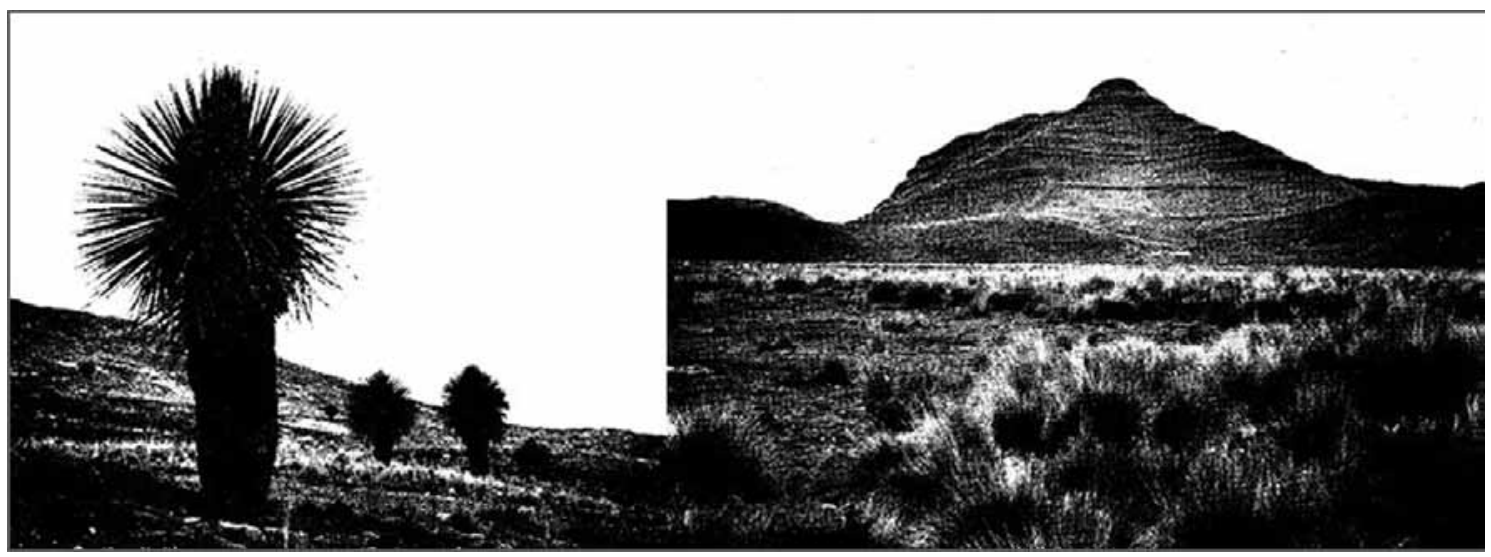

Rodal de puyas y pajonal de puna en la Hacienda Checayani (Fuente Dorst 1956) 
en Kiel en 1958-1959.

Entre las actividades realizadas en la estación de Checayani se encuentra el estudio y crianza de la chinchilla. Hernando De Macedo fue cofundador y director científico de la Chinchilla Fur Company, de 1963 a 1967.

Los estudios y actividades realizados en esta estación tuvieron situaciones difíciles. Durante la reforma agraria iniciada en 1969 la Hacienda Checayani fue afectada. Aproximadamente un tercio de ella, incluyendo el sector donde se encontraba la Estación Altoandina de Biología, permaneció como propiedad familiar y la estación continuó actividades durante un tiempo. Sin embargo, la irrupción de la actividad terrorista en los años 1980's obligó a la suspensión de su funcionamiento y el último científico visitante fue el Dr. Manfred Röhrs, quien en 1982 realizó estudios comparativos del cerebro de cuyes silvestres y do-

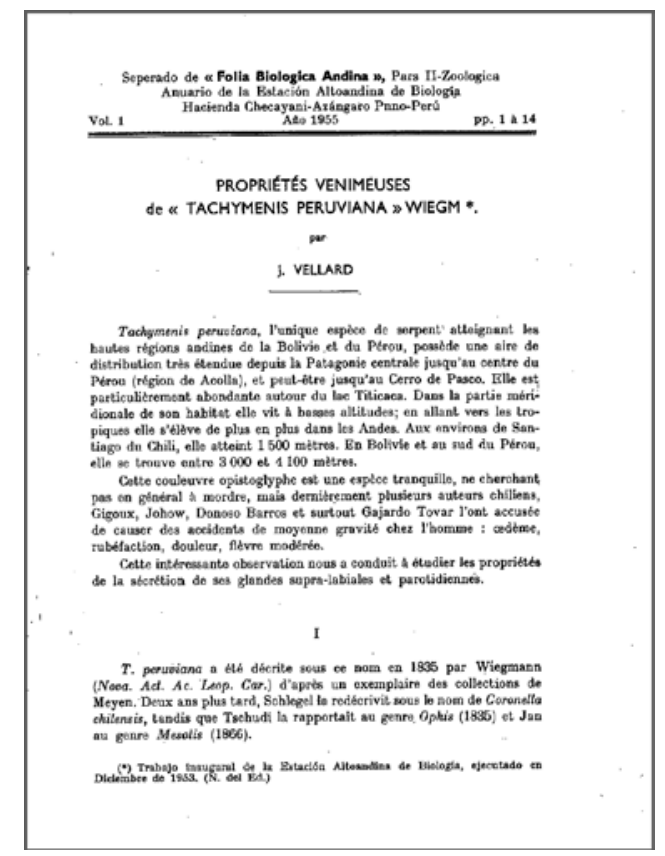

Primer Número de la Revista Folia Biologica Andina publicada por $\mathrm{H}$. de Macedo con el trabajo inaugural de la estación Altoandina de Biología de J. Vellard (1955) mésticos (Aguilar 1998b, Ebinger, De Macedo \& Röhrs 1984).

Sus esfuerzos en la Estación Altoandina de Biología fueron recompensados con varios reconocimientos. Debido a los logros obtenidos en esta estación andina recibió en 1956 la medalla de plata de la institución francesa Sociedad de Aclimatación y Protección de la Naturaleza y ese mismo año fue nombrado Oficial por la institución Orden Universal del Mérito Humano en Génova, Suiza.

\section{Folia Biológica Andina, Anuario de la Estación Altoandina de Biología, Hacienda Checayani, Azángaro, Puno y otras publicaciones}

Para la difusión de los resultados de los estudios realizados en la Estación Biológica Andina y áreas aledañas, Hernando De Macedo creó la revista Folia Biológica Andina, en la que se publicó un importante número de trabajos que incluyeron algunos estudios realizados en otras localidades. Las principales publicaciones corresponden a Cuatrecasas (1954), Vellard (1955, 1957, 1959, 1960, 1966), Lindberg (1955, 1957), Brehm (1957 y Cerrate (1957). Esta revista fue impresa en Francia en la imprenta lmprimerie Alençonnaise, Place Poulet-Malassls, Alençon (Orne).

Entre los trabajos realizados en la Estación Biológica Andina y áreas aledañas y publicadas en otras revistas destacan las de Dorst (1956a, 1956b, 1957a, 1957b, 1958, 1962, Roe \& Rees (1979), Hückinghaus (1961), Kosswig \& Villwock (1964) y Ebinger, De Macedo \& Röhrs (1984). Maria Koepcke comentó sobre este tema en una publicación de Peruanische Post, un diario limeńo en alemán. El conjunto representa un total aproximado de 50 trabajos (Aguilar 1998b).

\section{Actividades académicas en Lima}

Hernando De Macedo contrajo matrimonio con Gloria Pinelo en 1965. Los primeros años posteriores al matrimonio la pareja residió en la Hacienda Checayani. Luego regresaron a establecerse en Lima y él se dedicó a la docencia universitaria (Gloria Pinelo de Macedo com pers). Ejerció la docencia durante un largo periodo (1970-1990). Muchos años enseñó principalmente en la Universidad Nacional Mayor de San Marcos, aunque también dictó cursos en las Universidades Cayetano Heredia y

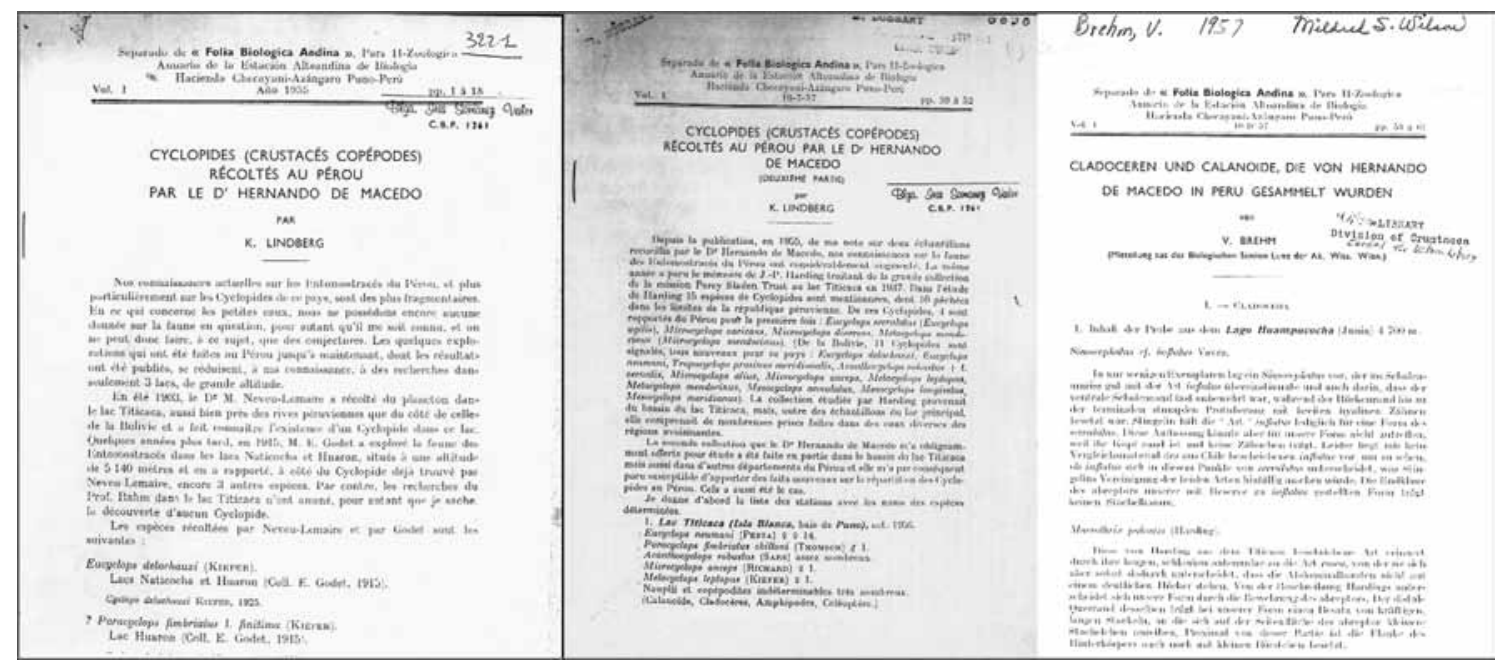

Trabajos de Lindberg 1955, 1957 y Brehm 1957 publicados en la revista Folia Biológica Andina, Anuario de la Estación Altoandina de Biología de Checayani. 


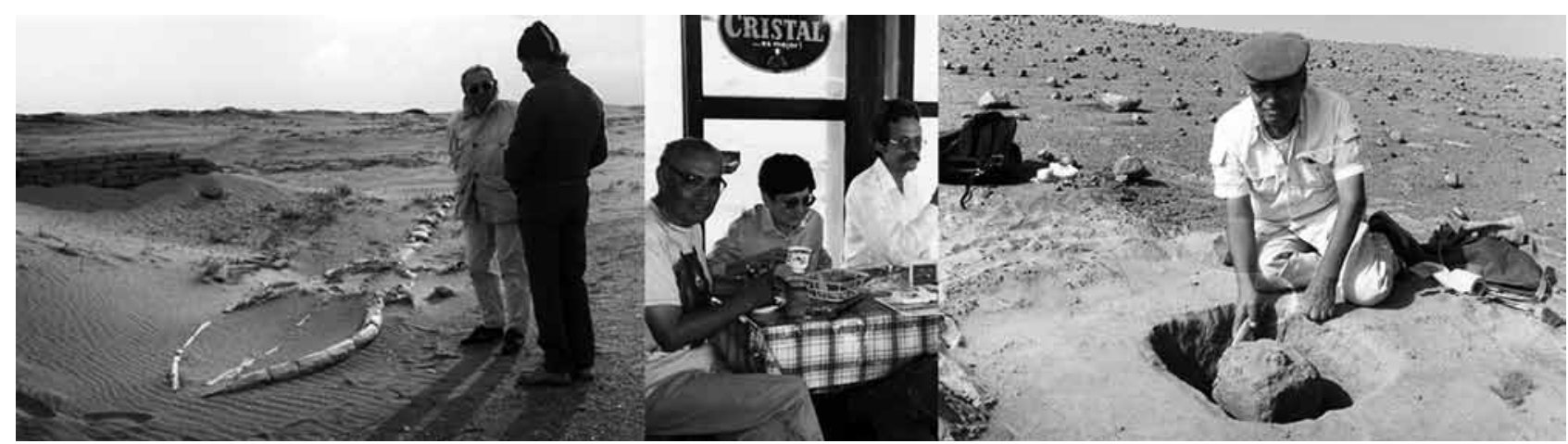

El Dr. Macedo participando en el trabajo de campo para recuperar fósiles en Sacaco, 1985 (Fotos P. Larson)

Ricardo Palma. Enseñaba los cursos de Anatomía Comparada de Cordados y Zoología de Cordados.

Aunque manifestaba con cierta frecuencia que no le agradaba la docencia, su curso era muy apreciado por sus alumnos. Muchos recuerdan sus clases, a las que llegaba portando varios libros (P. Huamán, com pers.). Mencionan que "con sus libros abiertos, escritos en alemán, inglés, francés, explicaba con detalle las tendencias sobre el tema de las distintas escuelas. Espectacular!!!! (Gabriel Ballón, com pers). Los exámenes de su curso eran orales. Hacía una pregunta y si el alumno no sabía, en vez de reprobarlo le volvía a explicar todo el tema hasta que lo entendiera y ambos estuvieran satisfechos (Franz Cardoso, com pers).

En reconocimiento a su labor fue nombrado Profesor Emérito de la Universidad Nacional Mayor de San Marcos en 2002, en el congreso Latinoamericano de Herpetología del 2002 en Lima se le rindió homenaje por sus contribuciones al estudio de anfibios y reptiles en Perú y fue nombrado Profesor Emérito de la Universidad Ricardo Palma en 2008.

\section{En el Museo de Historia Natural}

Hernando De Macedo estuvo muy ligado al Museo de Historia Natural desde sus años de estudiante. Se alejó de este durante varios periodos para ocuparse de la Estación Altoandina de Biología de Checayani y en sus viajes de estudios al extranjero.

Cuando en 1968 los Drs. Koepcke se trasladaron a Panguana, Maria Koepcke continuó con el cargo de Jefe de la Sección de Aves y Mamíferos del Museo de Historia Natural de la que se ocupaba durante sus visitas a Lima, mientras el Dr. Hernando De
Macedo quedaba encargado en Lima de esta sección durante sus ausencias. Luego del trágico fallecimiento de la Dra. Koepcke en diciembre de 1971, el Dr. De Macedo asumió la Jefatura de esta Sección, cargo que tuvo hasta la división de esta Sección en los Departamentos de Ornitología y Mastozoología. Él permaneció como Jefe del Departamento de Mastozoología hasta su jubilación.

\section{Redescubrimiento del Mono Choro de Cola Amarilla}

Hasta 1974, el mono choro de cola amarilla, Oreonax flavicauda, estaba representado en las colecciones científicas de museos por sólo cinco ejemplares, el último de los cuales había sido colectado en 1926. Para intentar determinar si esta especie todavía existía, se organizó una expedición al área donde habían sido obtenidos los últimos ejemplares (Chachapoyas, Mendoza y Pedro Ruiz Gallo, departamento de Amazonas), conformada por Russell A. Mittermeier, Hernando De Macedo y B. Anthony Luscombe. Esta expedición partió de Lima el 26 de abril de 1974. Cuando la expedición estaba dirigiéndose a Chachapoyas se encontró con un cazador que tenía una piel del mono choro de cola amarilla que había cazado seis días antes. Posteriormente este cazador proporcionó otras tres pieles. Sin embargo, durante los doce días que duró la expedición, no pudieron localizar ningún individuo silvestre. El día anterior a su regreso a Lima, los niños del pueblo llevaron a los tres expedicionarios a la vivienda de un soldado que tenía un bebé del mono choro de cola amarilla. Este fue inmediatamente comprado y llevado a Lima (Mittermeier, Macedo-Ruiz \& Luscombe 1977).

El joven mono choro de cola amarilla que recibió del nom-

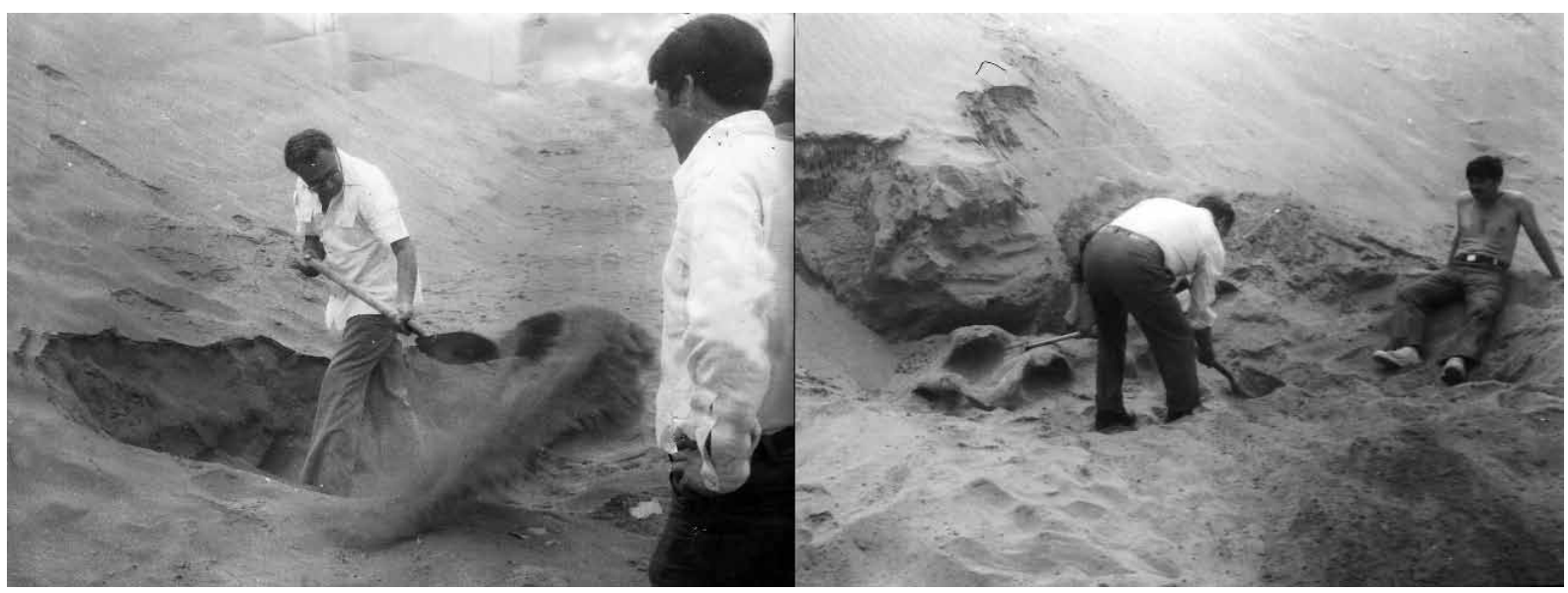

Colecta de una pelvis y un fémur de mastodonte en niveles pleistocénicos de la Escuela de Policía de Chorrillos en los años 1970's (Fotos R. Salas) 


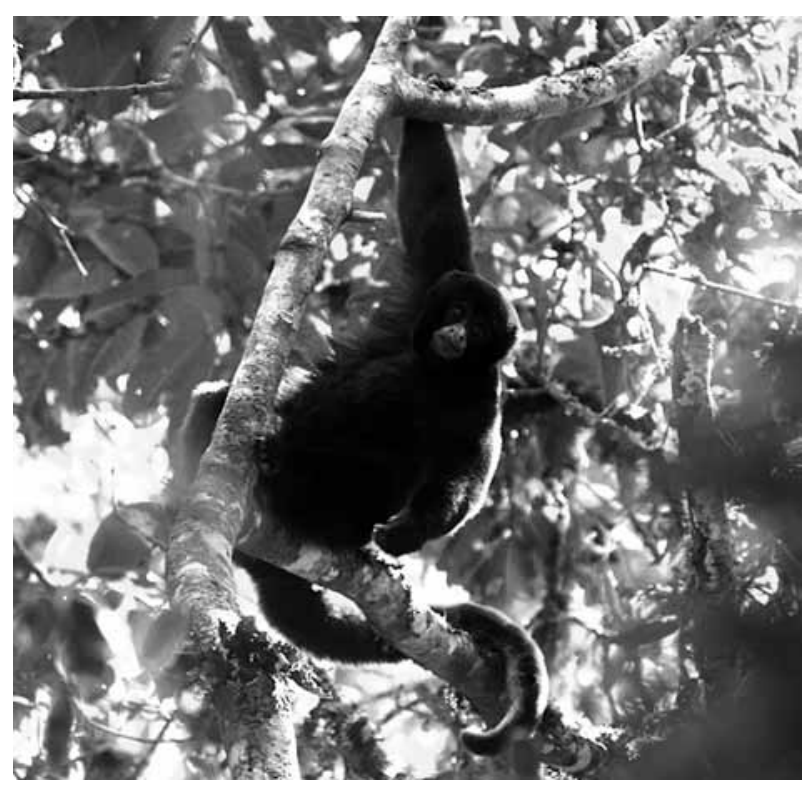

Mono choro de cola amarilla, Oreonax flavicauda, recescubierto por Russell A. Mittermeier, Hernando De Macedo y B. Anthony Luscombe en 1974 (Foto Wikipedia)

bre de "Flavi" y fue donado oficialmente al Museo de Historia Natural. Inicialmente fue criado durante un par de ańos en su domicilio por el Dr. De Macedo y su esposa Gloria junto a dos monos choros comunes (Lagothrix lagotricha) llamados "Petete" y "Chimpa" (Gloria Pinelo de Macedo com pers). Posteriormente "Flavi" y "Petete", fueron llevados a una jaula especialmente acondicionada en el jardín interior del Museo de Historia Natural, siempre bajo la tutela del Dr. De Macedo. Durante un par de ańos estos monos fueron apreciados por los escolares y público en general que visitaba el museo, así como apreciados y engreídos por los profesores y trabajadores de la institución. Al desarrollar como individuos adultos se volvió una tarea cada vez más difícil su mantenimiento en el museo y finalmente fueron transferidos al Parque de Las Leyendas.

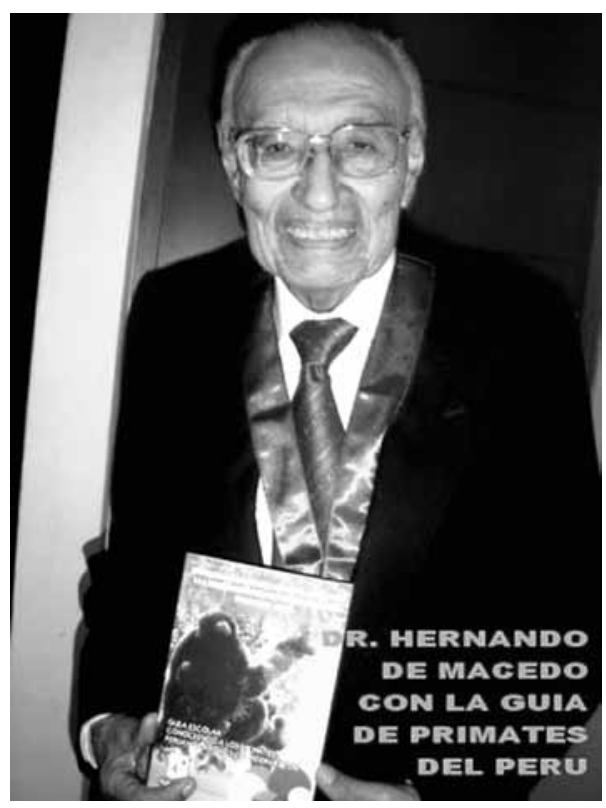

Dr. Macedo con la Guía de Primates del Perú (Fuente Humboldt Club del Perú.

\section{Primeros en observar Pavas Aliblancas vivas recién redescubiertas}

La pava aliblanca, Penelope albipennis, descrita por Taczanowski en 1877 , se conoció durante 100 años a través de tres pieles. El ejemplar tipo se encontraba en el museo de Varsovia, donde había trabajado Taczanowski. Un segundo ejemplar formaba parte de la exhibición de aves del Museo de Historia Natural de la UNMSM y era expuesto con una etiqueta que indicaba "EXTINTO". El tercer ejemplar se encontraba en el Museo Británico de Historia Natural. El Dr. De Macedo estuvo muy cercano a los acontecimientos del redescubrimiento de la pava aliblanca, el 13 de setiembre de 1977. Muy poco después de este suceso, él formó parte del pequeño grupo integrado además por el conservacionista Gustavo del Solar, re-descubridor de la pava aliblanca, y el fotógrafo Heinz Plenge, quienes viajaron inmediatamente después a la zona. El 27 y 28 de setiembre, ellos fueron los primeros en poder observar esta ave viva y fotografiarla. Estos sucesos y la discusión sobre la pava fueron publicados por el Dr. De Macedo en dos artículos (1979a, 1979b).

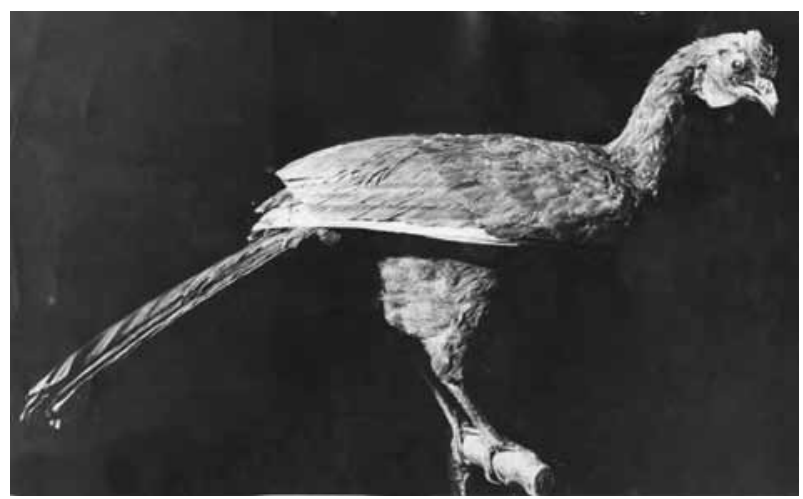

Fotografía de los años 60 del ejemplar de la pava aliblanca, Penelope albipennis, expuesta en las exhibiciones del Museo de Historia Natural como "EXTINTA" durante muchos años hasta su redescubrimiento en 1977. Hernando De Macedo formó parte del primer grupo de personas que pudieron verla viva pocos días después del redescubrimiento (Foto H. De Macedo).

\section{Director del Museo de Historia Natural}

Entre 1981 y 1985 el Dr. De Macedo fue Director del Museo de Historia Natural. Durante su gestión apoyó todas las iniciativas para mejorar el museo. Su prolongada afición a los primates luego del redescubrimiento del mono choro de cola amarilla y la crianza de "Flavi" lo impulsó a gestionar la financiación, a través de Russell A. Mittermeier, de la remodelación las exhibiciones de la sala de primates.

Recuerda también el botánico Kember Mejía que en diciembre de 1982 retornaba él de Iquitos con una gran carga de las primeras muestras botánicas de palmeras peruanas. En ese momento en el herbario solamente había fotografías en blanco y negro tomadas por Harold Moore. El Dr. De Macedo lo encontró justo en la entrada y al ver los voluminosos paquetes le pregunto de qué se trataba. Al enterarse del contenido y de la utilidad de estas plantas, con entusiasmo muy grande le indicó: ¡Puedes escribir algo sobre las palmeras?¡; ¡ Tenemos que hacerles 


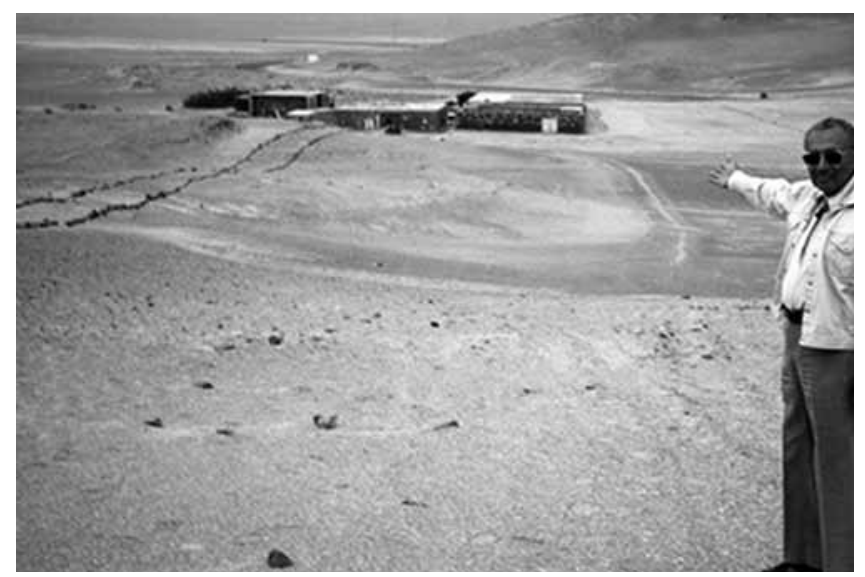

Hernando de Macedo indicando el Museo de Sitio de Sacaco, logrado durante su gestión como Director del Museo de Historia Natural de la UNMSM

un espacio para exhibirlas en el Museo; Y efectivamente le ayudó a publicar el folletito "Las palmeras y el selvícola Amazónico" y se adecuó una sala para exhibir partes de las especies más importantes.

Brindó también un decidido apoyo a proyectos de investigación, entre ellos el estudio del Bosque de Zárate que realizaban a inicios de los años 80 Niels Valencia y la autora. Su apoyo facilitó la obtención de financiación de la Sociedad Zoológica de Frankfurt para este proyecto.

Personalmente, la autora le debe un inmenso agradecimiento por acogerla en la Sección de Aves y Mamíferos y permitirle desarrollarse en ella para lo cual siempre contó con su incondicional apoyo.

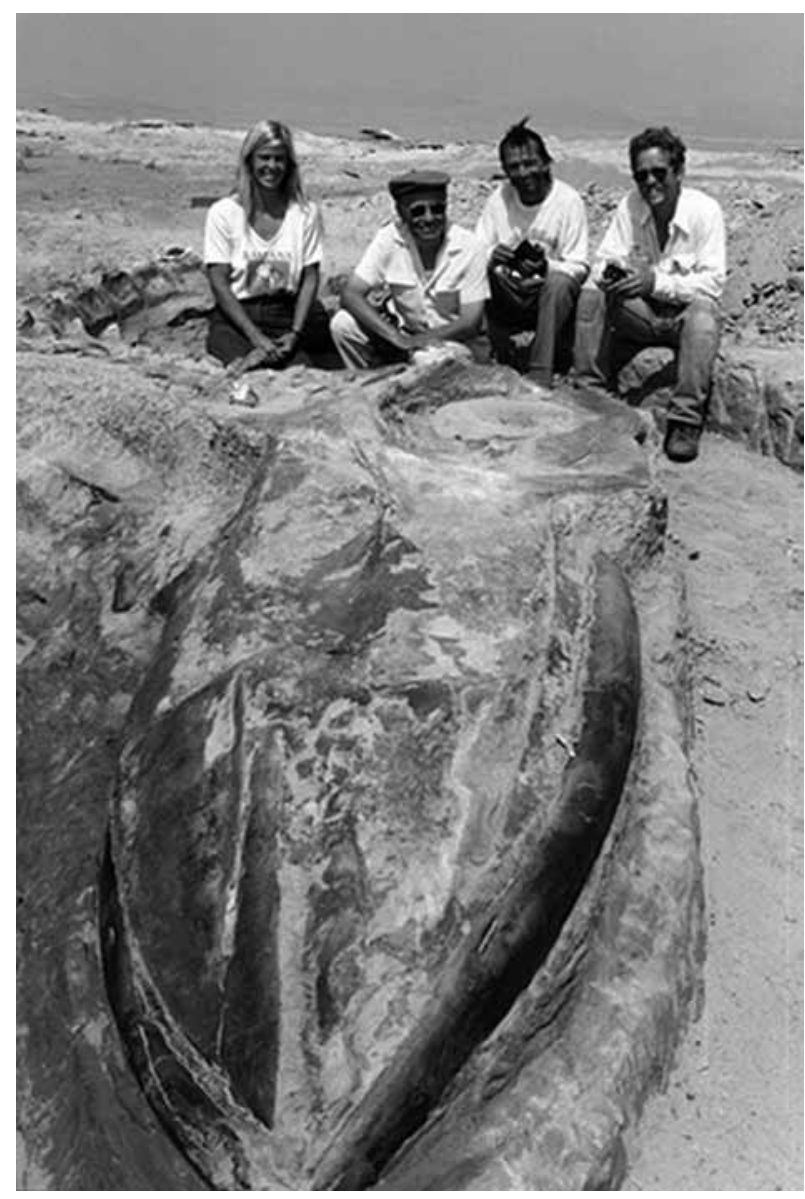

Hernando de Macedo con ballena fósil, Sacaco 1985

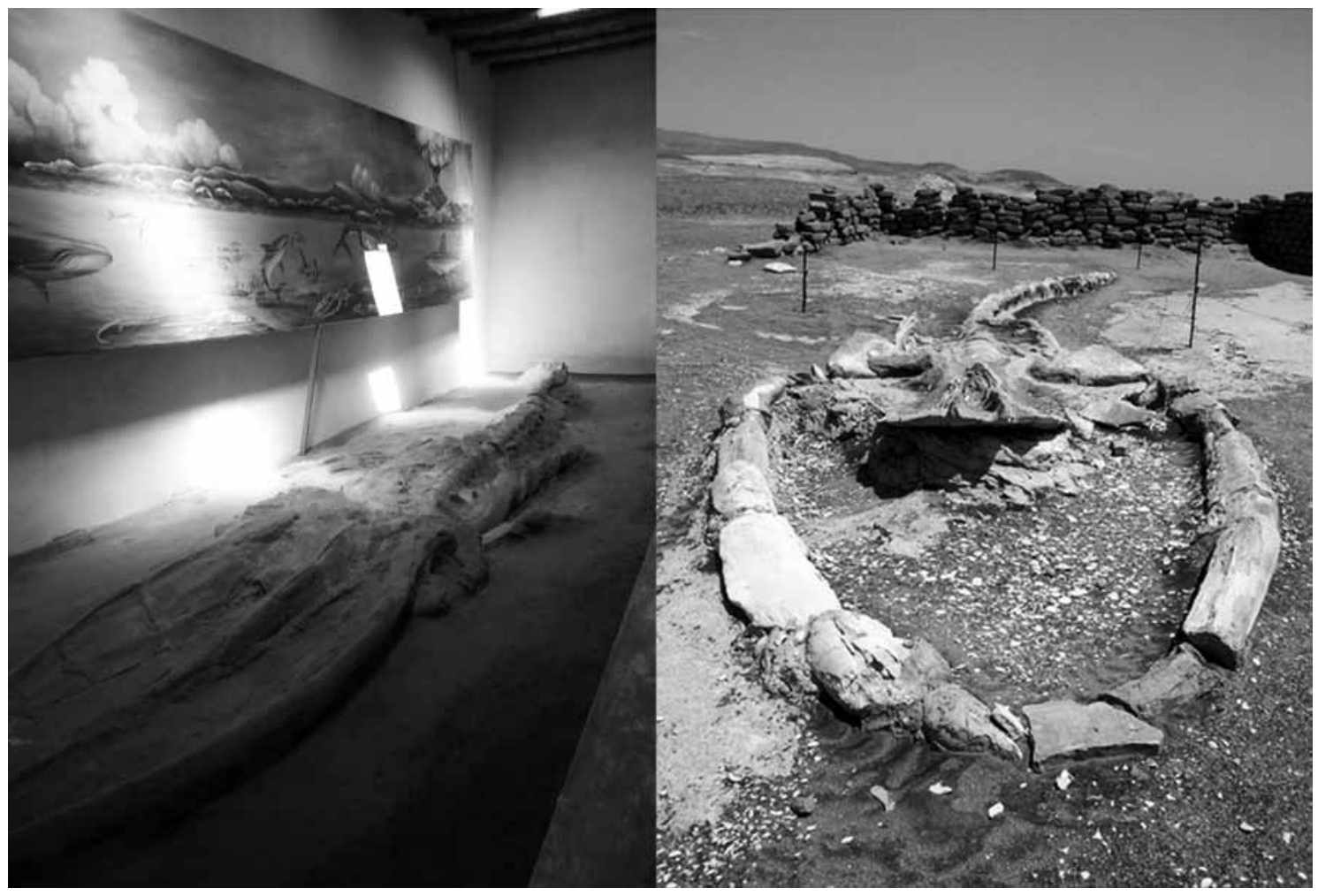

Ballenas fósiles en las exhibiciones interna y externa del Museo de Sitio de Sacaco (Fotos I. Franke) 


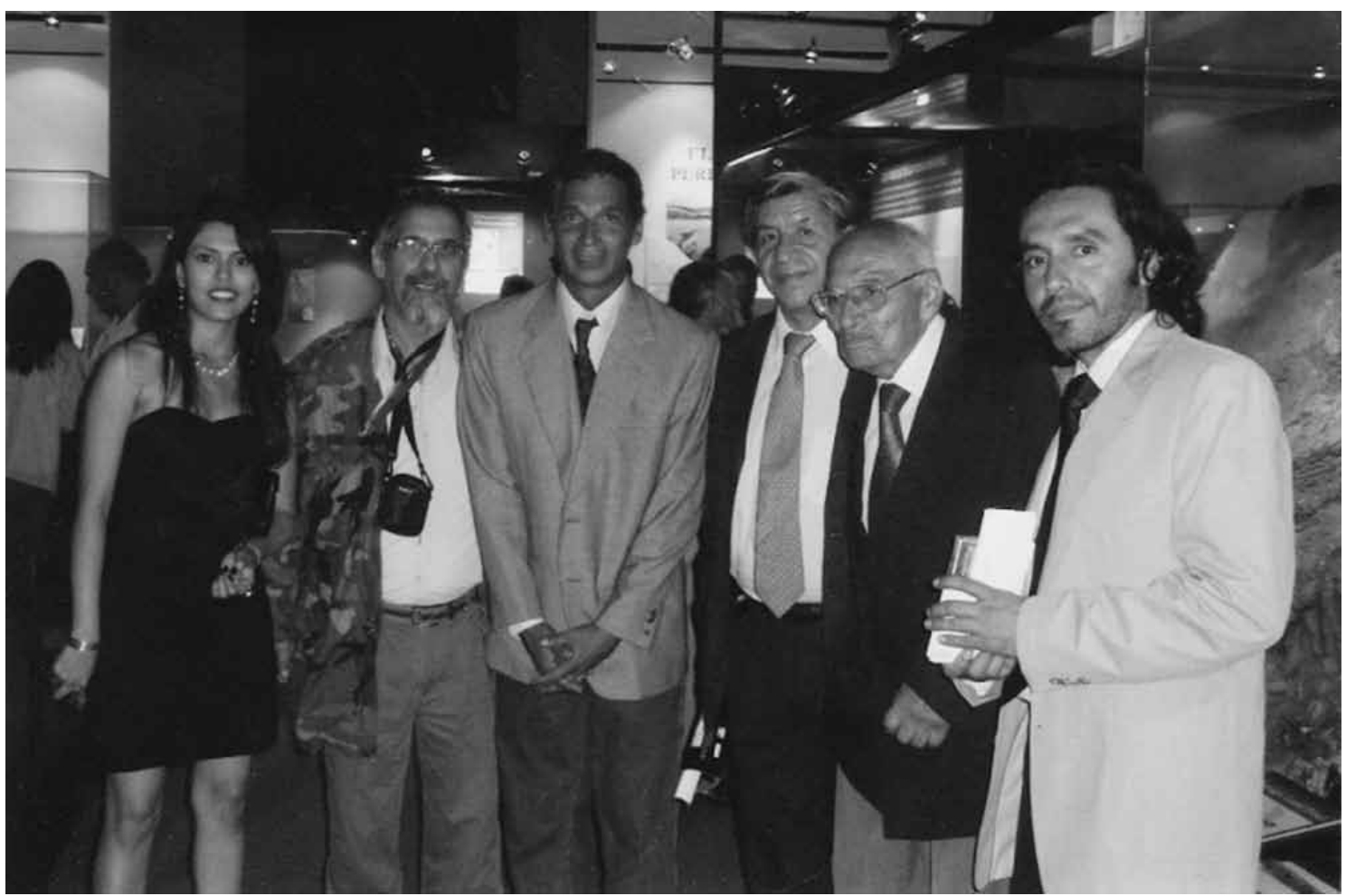

Inauguración de la remodelada sala de exhibición de la Formación Pisco, 2011 (Foto E. Salas).

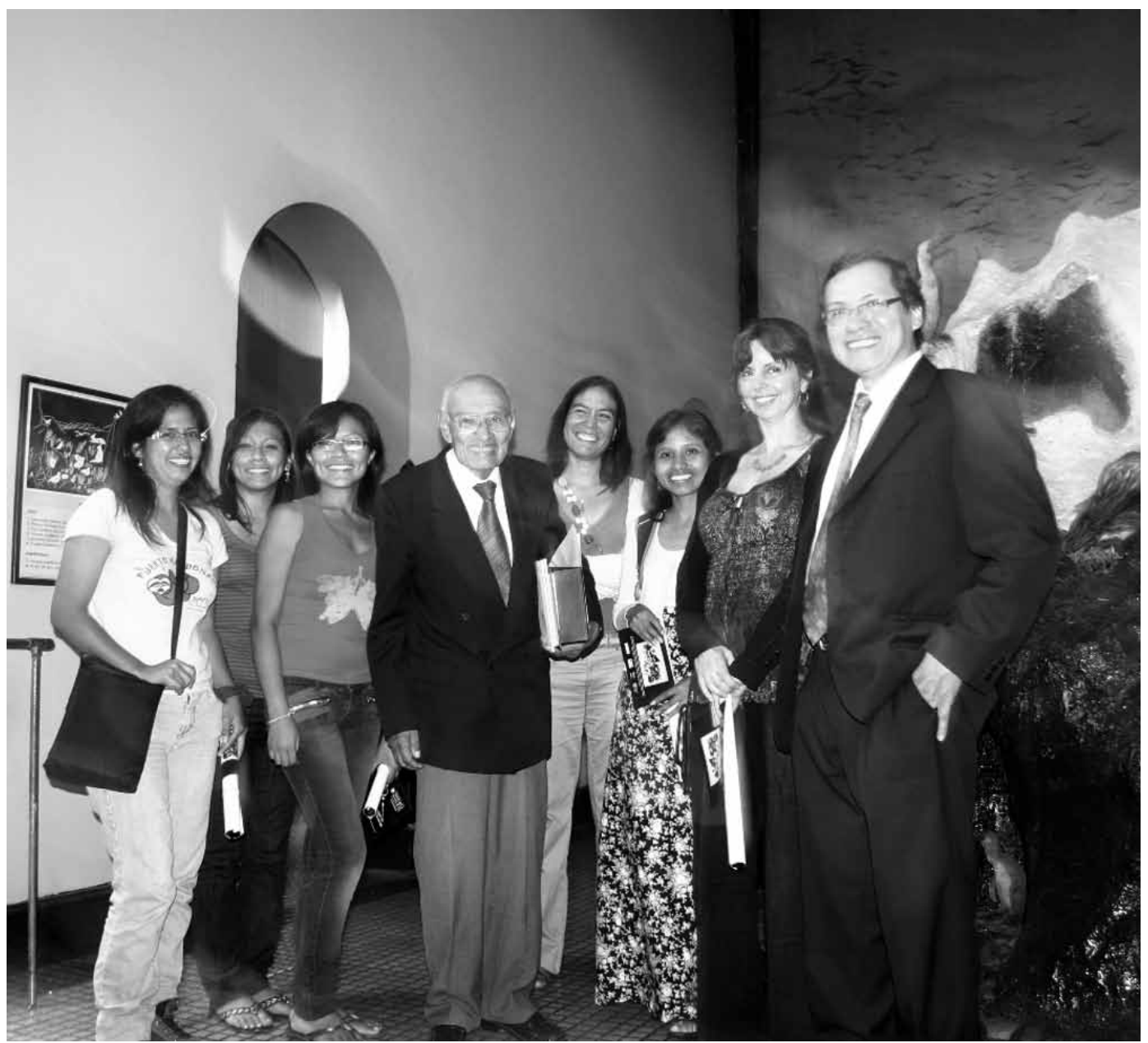

Celebrando la remodelación de la sala de mamíferos del Museo de Historia Natural, 28 febrero 2011 (Foto V. Pacheco) 


\section{Paleontología de Vertebrados}

Otro de los importantes aportes de su gestión como Director del Museo de Historia Natural, fue impulsar y dar importancia a la Paleontología de Vertebrados, área que no se trabajaba en el Perú y que ha desarrollado notablemente en los últimos años, dando a conocer la Mega-Biodiversidad del pasado en casi todos los grupos de fauna, incluyendo los pingüinos gigantes de nuestra costa (Franke 2014).

La afición del Dr. De Macedo por los fósiles tenía una prolongada historia que muy probablemente estaba relacionada con su excelente preparación en anatomía comparada. Ya en los ańos 70 colectó una pelvis y un fémur de mastodonte en niveles pleistocénicos de la Escuela de Policía de Chorrillos. Siendo Jefe de la Sección de Aves y Mamíferos del Museo de Historia Natural, tuvo bastante contacto con paleontólogos de vertebrados extranjeros que trabajaban en el Perú, entre ellos Kenneth E. Campbell.

Durante su gestión como Director del Museo, tuvo la oportunidad de proveer a las exhibiciones del Museo del primer ejemplar de una ballena fósil traído de los ricos yacimientos de fósiles de Sacaco. El participó del trabajo de campo realizado por Peter Larson para recuperar el fósil, al que todos se referían afectuosamente como "Hernando". También gracias a su gestión se construyó el Museo de Sitio de Sacaco, ubicado en el km 546 de la carretera Panamericana Sur y que tiene como exhibiciones principales fósiles de ballenas tanto en el interior de la instalación como en una presentación cercana al aire libre. Este museo es una filial del Museo de Historia Natural de la UNMSM. La exhibición de la ballena fósil y el Museo de Sitio de Sacaco fueron financiados por Hans Jacob Siber.

Recuerda Rodolfo Salas, actual encargado del Departamento de Paleontología de Vertebrados, que cuando él era niño el Dr. De Macedo lo recibía amablemente en su laboratorio del Museo de Historia Natural y se tomaba el tiempo para mostrarle los fósiles que había descubierto y explicarle con paciencia sobre sus características. Indudablemente estas experiencias contribuyeron a que R. Salas se interesara en el campo de la paleontología y sea en la actualidad uno de los principales líderes del desarrollo de este campo en el Perú. Posteriormente, ya como investigador emérito de la universidad, el Dr. De Macedo fue un inestimable consejero y amigo de los miembros de este departamento, donándoles parte de su biblioteca personal.

Posteriormente a su jubilación, el Dr. De Macedo fue invitado a dar varias conferencias en el Museo de Historia Natural, donde era muy apreciado pues había destacado por su caballerosidad, amabilidad y generosidad. Mantuvo la costumbre de realizar visitas esporádicas al museo hasta su fallecimiento acaecido en Lima el 31 de marzo de 2015.

Como testimonia Victor Pacheco, Hernando De Macedo ha dejado una profunda huella en sus alumnos y colegas por su excelente calidad humana y docente, su paciencia para abordar y enseñar con profundidad complejas estructuras anatómicas, su capacidad de asombro ante los descubrimientos, su generosidad para compartir su biblioteca especializada y sus vastos conocimientos sobre prácticamente cualquier vertebrado del Perú.

\section{Agradecimientos}

Deseo expresar mi profundo agradecimiento a Rodolfo Salas, Victor Pacheco, Gloria Pinelo de Macedo, Julia Tejada, Mario Rosina, Iris Samanez, Cesar Aguilar, Mikael Lundberg, Peter Larson, Gabriel Ballón, Franz Cardoso y el Dr. Carlos Paredes por compartir sus recuerdos del Dr. De Macedo y por los materiales que facilitaron para este artículo.

\section{Literatura citada}

Aguilar F., P. G. 1998a. El Museo de Historia Natural de la Universidad Nacional Mayor de San Marcos y la enseñanza de las Ciencias Biológicas. Publicación con motivo de las Bodas de Oro de la Promoción 1948 Javier Ortiz de la Puente Denegri.

Aguilar F., P. G. 1998b. La Estación Altoandina de Biología y Reserva Zoobotánica de Checayani. Publicación con motivo de las Bodas de Oro de la Promoción 1948 Javier Ortiz de la Puente Denegri.

Franke, I. 2014. Pingüinos gigantes en la costa peruana. Blog Aves, Ecología y Medio Ambiente, 19 noviembre 2014 http:// avesecologaymedioambiente.blogspot.com/2014/11/ pinguinos-gigantes-en-la-costa-peruana.html

USAID. 1966. Basic data and background information for the workshop on the role of science and technology in Peruvian economic developtment held in Paracas, Peru, April 1722, 1966. Office of the Foreign Secretary of the National Academy of Sciences in collaboration with the Agency for International Development.

\section{Publicaciones del Dr. Hernando De Macedo}

De Macedo, H. 1950. Anotaciones para el conocimiento zoológico del género Batrachophrynus (Amphibia: Salientia). Tesis universitaria para optar el Título de Bachiller. Universidad Nacional Mayor de San Marcos, Lima. 27 p.

Macedo R., Hernando de. 1964. Curieux cas de nidification du Buteo poecilochrous Gurney sur Puya raimondii. L' Oiseau et la Revue Française d'Ornithologie, 34: 200-203.

Mittermeier, R.A.; Macedo Ruiz, H.; Luscombe, A. 1975. A Woolly Monkey Rediscovered in Peru. Oryx, 13(01): 41-46.

De Macedo, H. 1976. Aspectos ecomorfológicos de los aparatos respiratorios y circulatorios en anfibios andinos. Tesis universitaria para optar el título de Doctor en Ciencias. Universidad Nacional Mayor de San Marcos, Lima. 51 p.

Mittermeier, R.A., Macedo-Ruiz, H. de, Luscombe, B.A.y Cassidy, J. (1977) Rediscovery and conservation of the Peruvian yellowtailed woolly monkey (Lagothrix flavicauda) Rev. Cienc. 71.

Macedo R., Hernando de. 1978. Redécouverte du Cracidé Penelope albipennis dans les forêts sèches du nord-ouest du Pérou. C. R. Acad. Sc. Paris, 287 (Série D): 265-267. [Possibly Comptes rendus hebdomadaires des séances de l'Académie des sciences. Série D: Sciences Naturelles, Paris]

Macedo R., Hernando de. 1979a. Redescubrimiento de la Pava Aliblanca Penelope albipennis Taczanowski 1877. Boletín de Lima, no. 1: 5-11.

Macedo R., Hernando de. 1979b. 'Extinct' bird found in Peru. Oryx, 15: 33-37.

Macedo-Ruiz, H. De; Mittermeier, R.A. 1979. Redescubrimiento del primate peruano Lagothrix flavicauda (Humboldt 1812) y primeras observaciones sobre su biología. Revista de Ciencias de la Universidad Nacional Mayor San Marcos 71: 78-92.

Ebinger, P., de Macedo, H., Röhrs, M., 1984. Hirngrößenänderung vom Wild- zum Hausmeerschweinchen. Z. Zool. Syst. Evol.-forsch. 22, 77-80.

Macedo R., H. de 1998. Javier Ortiz de la Puente Denegri. Biólogo UNMSM, Promoción 1948. (1928-1952). Publicación con motivo de las Bodas de Oro.

\section{Folia Biológica Andina}

Cuatrecasas, J. 1954. El género Mniodes. Folia Biologica Andina, $1: 1-7$, Puno 
Vellard, J. 1955. Propriétés venimeuses de Tachymenis peruviana Wiegm. Folia Biologica Andina, Pars II - Zoologica 1: $1-14$.

Lindberg, K. 1955. Cyclopides (crustaces copepodes) recoltes au Perou par le Dr Hernando de Macedo. Folia Biologica Andina, Pars II, Zoologica 1:1-18.

Lindberg, K. 1957. Cyclopides (Crustacés, Copepodés) récoltés au Pérou par le Dr. Hernando de Macedo. (Deuxième Partie). Folia Biologica Andina, Part 2, Zoología, Anuário de la Estación Altoandina de Biología, Hacienda ChecayaniAzangaro, Puno, Peru. Volume: 1:39-52.

Vellard, J. 1957. El Grupo Gastrotheca. Folia Biologica Andina 4: 48p.

Brehm, V. 1957. Cladoceren und Calanoiden, die von Hernando De Macedo in Peru gesammelt wurden. Folia Biologica Andina, Part 2, Zoología, Anuário de la Estación Altoandina de Biología, Hacienda Checayani-Azangaro, Puno, Peru 1:53-61.

Cerrate, E. 1957. Notas sobre la Vegetación del Valle de Chiquián. Folia Biologica Andina 1: 9-39

Vellard, J. 1959. El Género Bufo. Folia Biologica Andina 5: 48p.

Vellard, J. 1960. Notas complementarias sobre Telmatobius. Folia Biologica Andina 6: 18p.

Vellard, J. 1966. El género Peurodema en los Andes. Folia Biologica Andina 7: 12p.

\section{Trabajos realizados en la en la Estación Bioló- gica Andina y áreas aledañas, y publicadas en otras revistas (no Folia Biológica Andina)}

Dorst, J. 1956a. Étude biologique des Trochilidés des hauts plateaux péruviens. L'Oiseaux et R. F. O. 26: 165-193

Dorst, J. 1956b. Étude d'une collection d'oiseaux rapportée des hauts plateaux andins du Pérou méridional. Bulletin du Muséum National d'Histoire Naturelle, Paris. (2) 28: 435-445.

Dorst, J. 1957a. Étude d'une collection d'oiseaux des hauts plateaux andins du Pérou méridional. Bulletin du Muséum National d'Histoire Naturelle, Paris, (2) 29: 127-129.

Dorst, J. 1957b. The Puya stands of the Peruvian high plateaux as a bird habitat. Ibis, 99: 594-599

Dorst, J.1958. Contribution a l'etude ecologique des rongeurs des haut plateaux du Pérou meridional. Mammalia 22:547-565

Dorst, J.1962a. A propos de la nidification hypogée de quelques oiseaux des hauts Andes péruviennes. L'Oiseau et la Revue Française d'Ornithologie, 32: 5-14.

Dorst, J. 1962b. Étude d'une collection d'oiseaux rapportée des hautes Andes méridionales du Pérou. Bulletin du Muséum National d'Histoire Naturelle, Paris, (2) 34: 427-434. http://www. bhl-europe.eu/static/a089323s/a089323s_full_pdf.pdf

Dorst, J 1972. Poids relatif du coeur de quelques rongeurs des hautes Andes Peruviennes. Mammalia. Volume 36, Issue 3, Pages 389-394,

Roe, N. A., and W. W. Rees. 1979. Notes on the puna avifauna of Azangaro Province, Department of Puno, southern Peru. Auk, 96:475-482.

Hückinghaus, F. 1961. Vergleinchende Untersuchungen über die Formenmannigfaltigkeit der Unterfamilie Caviinae Murray 1886. (Ergebnisse der Südamerikaexpedition Herre/Röhrs 1956-1957). Zeitschrift für Wissenschaftliche Zoologie 166: 1-98.

Kosswig, C. y W. Villwock., 1964. Das Problem der intralakustrischen Speziation im Titicaca und Lanaosse. Verh Dt. Zool. Ges. Kiel. 1964 Zool. Anz. Suppl. 28. 